\title{
Non-uniqueness of solutions of nonlinear heat equations of fast diffusion type
}

\author{
by \\ Ana RODRIGUEZ \\ Dpto. Matemática Aplicada E.T.S. Arquitectura \\ Univ. Politécnica de Madrid, 28040 Madrid, Spain. \\ and \\ Juan Luis VAZQUEZ \\ Departamento de Matemáticas Univ. Autónoma de Madrid, 28049 Madrid, Spain.
}

\begin{abstract}
We study the existence of infinitely many solutions for the Cauchy problem associated with the nonlinear heat equation $u_{t}=\left(u^{m-1} u_{x}\right)_{x}$ in the fast diffusion range of exponents $-1<m \leq 0$ with initial data $u_{0} \geq 0, u_{0} \not \equiv 0$. The issue of non-uniqueness arises because of the singular character of the diffusivity for $u \approx 0$. The precise question we want to clarify is: can we have multiple solutions even for initial data which are far away from the singular level $u=0$, for instance for $u_{0}(x) \equiv 1$ ? The answer is, rather surprisingly, yes. Indeed, there are infinitely many solutions for every given initial function. These properties differ strongly from other usual types of heat equations, linear or nonlinear.
\end{abstract}

We take as initial data an arbitrary function in $L_{\text {loc }}^{1}(\mathbf{R})$. We prove that when the initial data have infinite integral on a side, say at $x=\infty$, then we can choose either to have infinite mass for all small times at least on that side, and the choice is then unique, or finite mass, and then we need to prescribe a flux function with diverging integral at $t=0$, being otherwise quite general. Moreover, a new parameter appears in the solution set. The behaviour on both ends, $x=\infty$ and $x=-\infty$ is similar and independent.

Key words: Nonlinear heat equation, fast diffusion, nonuniqueness, Bäcklund transform.

Both authors partially supported by DGICYT Project PB90-0218.

A.M.S. Classification : $35 \mathrm{~K} 55,35 \mathrm{~K} 65$.

Annales de l'Institut Henri Poincaré - Analyse non linéaire - 0294-1449

Vol. $12 / 95 / 02 / \$ 4.00 /$ 
RÉSUMÉ. - Nous étudions l'existence d'une infinité de solutions pour le problème de Cauchy associé à l'équation de la chaleur non linéaire $u_{t}=\left(u^{m-1} u_{x}\right)_{x}$ dans le rang d'exposants $-1<m \leq 0$, avec donnée initiale $u_{0} \geq 0$ et non triviale. La possibilité de non-unicité apparaît à cause de la singularité de la diffusivité $u^{m-1}$ pour $u \sim 0$. La question précise que nous voulons clarifier peut être formulée comme suit : est-il possible d'avoir une multiplicité de solutions même pour une donnée initiale qui est partout loin du niveau singulier $u=0$ ? La réponse (plutôt surprenante) est OUI, il y a toujours non unicité, et même une infinité de solutions pour chaque donnée initiale $u_{0}$. Ces propriétés diffèrent notablement de celles des autres équations de la chaleur linéaires ou non linéaires qu'on a étudiées.

On prend des données initiales $u_{0} \in L_{\text {loc }}^{1}(\mathbf{R})$. Quand l'intégrale de $u_{0}$ diverge en $x=+\infty$ on a ou bien le choix d'une masse infinie de ce côté pour tout temps petit (et ce choix est unique) ou bien d'une masse finie toujours, et alors on a besoin d'imposer une fonction de fluxe $f(t)$ à l'infini avec intégrale divergente en $t=0$, autrement arbitraire. L'ensemble des solutions montre encore un paramètre supplémentaire. Le comportement pour $x=-\infty$ est similaire et indépendant.

\section{O. INTRODUCTION}

In this paper we investigate the existence of infinitely many solutions $u=u(x, t)>0$ of the fast-diffusion equation

$$
u_{t}=\left(u^{m-1} u_{x}\right)_{x}, \quad \text { for } \quad-1<m \leq 0,
$$

posed in $Q=\{(x, t): x \in \mathbf{R}, t>0\}$, taking initial data

$$
u(x, 0)=u_{0}(x), \quad x \in \mathbf{R},
$$

where $u_{0} \in L_{\text {loc }}^{1}(\mathbf{R}), u_{0} \geq 0$. The nonlinear evolution equation (0.1) has been proposed in several physical applications in the range of exponents $m<1$ as a model of diffusive phenomena where the diffusivity tends to infinity as $u \rightarrow 0$, thus receiving the name of fast-diffusion equation. Thus, the case $m=0$ (i.e. $u_{t}=(\log u)_{x x}$ ) appears for instance in the expansion of a thermalized electron cloud (Lonngren and Hirose [LH]), in gas kinetics (Carleman's model of Boltzman equation, Carleman [C], McKean [MK]), in ion exchange kinetics (Hellfrich and Plesset [HP]). Recently, Chayes, Osher and Ralston [COR] studied equation $(0.1)$ for $m \leq 0$ in connection with self-organized critical phenomena. 
The first mathematical studies concerned the range $0<m<1$, and it was proved that the Cauchy Problem is well-posed in $L^{1}(\mathbf{R})$, just as it happens in the classical heat equation, i.e. in the case $m=1$, and in the porous medium equation, $m>1$. However, this is not the case when $m \leq 0$. Indeed, the authors proved in [RV1] that in the present range $-1<m \leq 0$ The Cauchy Problem with initial data in $L^{1}(\mathbf{R})$ admits infinitely many solutions. More precisely, a well-posed problem can be obtained if we supplement $(0.1)-(0.2)$ with flux data at infinity. For every fixed $t>0$ we impose the conditions

$$
\begin{aligned}
& u^{m-1} u_{x} \rightarrow-f(t) \quad \text { as } \quad x \rightarrow \infty, \\
& u^{m-1} u_{x} \rightarrow g(t) \quad \text { as } \quad x \rightarrow-\infty .
\end{aligned}
$$

Given $f, g \in B V(\mathbf{R}), f, g \geq 0$, Problem (0.1)-(0.4) admits a unique solution $u \in C^{\infty}\left(Q_{T}\right)$ where $Q_{T}=\mathbf{R} \times(0, T)$ for some time $T>0$.

It is also proved in [RV1] that the "Neumann data" $f$ and $g$ cannot take negative values. Since the usual comparison theorems are valid, it follows that when $f$ and $g$ vanish identically we obtain the maximal solution of the problem. This solution has been investigated in an earlier paper with J. R. Esteban [ERV] and has special properties which it shares with the unique solution of the Cauchy Problem when $m>0$. A complete characterization of maximal solutions for general data measures is done in [RV2].

The aim of this paper is to clarify the existence of multiple solutions for the Cauchy Problem in the case when the initial data $u_{0}$ is not integrable, but merely locally integrable function, i.e. $u_{0} \in L_{\text {loc }}^{1}(\mathbf{R}), u_{0} \notin L^{1}(\mathbf{R})$. Some results are already known for this problem. The existence of a maximal solution $u \in C\left([0, \infty): L_{\text {loc }}^{1}(\mathbf{R})\right)$ which is $C^{\infty}$-smooth in $Q$ is also shown in [ERV]. Nonmaximal solutions appear in the literature in the form of travelling waves

$$
u(x, t)=f(x+c t), \quad c>0,
$$

which satisfy the following side conditions for $t>0$ :

$$
\begin{aligned}
& u \rightarrow 1 \text { as } x \rightarrow \infty, \\
& u^{m-1} u_{x} \rightarrow k \text { as } x \rightarrow-\infty .
\end{aligned}
$$

Such problems have been investigated by several authors, like Takáč [T1], [T2], Zhang [Z] and Chayes et al. [COR]. Self-similar solutions are also constructed by van Duijn, Gomes and Zhang [DGZ]. 
All the above-mentioned cases of non-uniqueness concern solutions which approach the "singular level" $u=0$. It could be conjectured that data which stay away from that level, like $u_{0}(x) \equiv 1$, would not admit multiple solutions. In fact, the main purpose of this work is to show that such assertion is always false, and more precisely, that for every nontrivial initial data $u_{0} \in L_{\text {loc }}^{1}(\mathbf{R}), u_{0} \geq 0$, there are infinitely many solutions of the Cauchy problem, corresponding in particular to the prescription of flux data (as in $(0.3),(0.4)$ ), subject only to certain compatibility and regularity conditions. Even more, in cases of infinite initial mass we discover the existence of an additional free parameter than can be prescribed.

It is interesting to remark that for bounded initial data we construct infinitely many bounded solutions of the Cauchy problem, a radical deviation from the behaviour of the equation for $m>0$, in particular of the classical heat equation.

Our study is complemented by showing the uniqueness of the solution which corresponds to zero-flux prescription, which coincides with the maximal solution. Uniqueness also holds for cases of finite initial mass on one side with the corresponding flux prescription on that side. Further information and comments are given in the final Section 7.

\section{PRELIMINARIES. GENERAL CONSIDERATIONS}

According to the theory developped in [RV1] we can solve the mixed initial and boundary-value Problem $(0.1)-(0.4)$ for every nonnegative initial data $u_{0} \in L^{1}(\mathbf{R})$ and every pair of flux functions $f$ and $g$ which are nonnegative and belong to $L^{1}(0, \infty) \cap B V_{\text {loc }}(0, \infty)$. The solution is a positive and $C^{\infty}$-smooth function defined in a strip $Q_{T}=\mathbf{R} \times(0, T)$ for some $T>0$. We have $u \in C\left([0, T]: L^{1}(\mathbf{R})\right)$, and the following formula holds

$$
\int_{\mathbf{R}} u(x, t) d x=\int_{\mathbf{R}} u_{0}(x) d x-\int_{0}^{t}(f+g) d t
$$

It is customary to refer to the integral $\int u(x, t) d t=M(t)$ as the mass of the solution at time $t$, and (1.1) is then called the global mass balance. 
The formula is valid for all times $t>0$ if $\int_{\mathbf{R}} u_{0}(x) d x$ is larger than $\int_{0}^{\infty}(f+g) d t$. Otherwise, there exists a time at which the second member of (1.1) becomes 0 . This time is given by

$$
T=\sup \left\{t>0: \int u_{0}(x) d x>\int_{0}^{t}(f+g) d t\right\}
$$

If $T$ is finite our solution vanishes identically as $t \rightarrow T$, and the equation ceases to have a meaning for $t \geq T$. Such $T$ is called the extinction time.

A very interesting property of the mixed problem $(0.1)-(0.4)$ is the stability property which can be stated as follows. Let $u_{i}, i=1,2$ be two solutions with initial data $u_{0 i}$ and flux functions $f_{i}, g_{i}$, all of them satisfying the properties stated above. Then

$$
\begin{aligned}
\int_{\mathbf{R}}\left(u_{1}(t)-u_{2}(t)\right)_{+} d x \leq & \int_{\mathbf{R}}\left(u_{01}-u_{02}\right)_{+} d x \\
& +\int_{0}^{t}\left\{\left(f_{2}-f_{1}\right)_{+}+\left(g_{2}-g_{1}\right)_{+}\right\} d t
\end{aligned}
$$

as long as both solutions exists. As a consequence, we have the following Maximum Principle: If $u_{01} \leq u_{02}, f_{2} \leq f_{1}$ and $g_{2} \leq g_{1}$, then $T_{1} \leq T_{2}$ and $u_{1} \leq u_{2}$ in $Q_{T_{1}}$.

The study of uniqueness is performed in [RV1] by means of the "potential function"

$$
U(x, t)=\int_{-\infty}^{x} u(x, t) d x+\int_{0}^{t} g(t) d t
$$

Using the fact that $u(x, t)$ is a solution of equation $(0.1)$, this potential can be written equivalently in the form:

$$
U(x, t)=\int_{\gamma} u d x+u^{m-1} u_{x} d t
$$

integrated along any curve $\gamma$ lying in $\bar{Q}$ and joining the points $(-\infty, 0)$ and $(x, t)$, since in (1.4) we are integrating an exact differential. It is explained Vol. $12, n^{\circ} 2-1995$. 
in $[\mathrm{RV} 1]$ that $U(x, t)$ is the unique solution of the problem

$$
\left\{\begin{array}{l}
U_{t}=\left|U_{x}\right|^{m-1} U_{x x} \\
U(+\infty, t)=\int_{-\infty}^{+\infty} u(x, t) d x+\int_{0}^{t} g d t \\
\quad=\int_{-\infty}^{+\infty} u_{0} d x-\int_{0}^{t} f d t \\
U(-\infty, t)=\int_{0}^{t} g(t) \\
U(x, 0)=\int_{-\infty}^{x} u_{0}(x) d x .
\end{array}\right.
$$

Arguments involving $U$ will be frequent in the sequel. When the integral $\int_{-\infty}^{0} u_{0} d x$ is infinite we shift the origin of $\gamma$ from $x=-\infty$ to $x=0$.

Let us now comment on our present project. We want to describe the solutions of the Cauchy Problem (0.1)-(0.2) for general initial data in $L_{\text {loc }}^{1}(\mathbf{R}), u_{0} \geq 0$. Therefore, we have to consider solutions with infinite initial mass, i.e. with $\int u_{0} d x=\infty$. Though the general results will have a unified outlook, it will be convenient to divide the study into a number of cases as follows.

Firstly, the behaviour at both ends, $x \rightarrow \infty$ and $x \rightarrow-\infty$ turns out to be independent in a sense. Therefore, we may consider two different initial situations: (i) when the mass is infinite only at one end, say, when

$$
\int_{-\infty}^{0} u_{0} d x<\infty \quad \text { and } \quad \int_{0}^{\infty} u_{0} d x=\infty
$$

(the simply infinite case) and (ii) when both integrals are divergent (the doubly infinite case). We will also need to consider independently the two partial mass functions

$$
M_{+}(t)=\int_{0}^{\infty} u(x, t) d x, \quad M_{-}(t)=\int_{-\infty}^{0} u(x, t) d x .
$$

There are then two possibilities to be considered for a solution which has an infinite initial mass on one side. Either (a) it stays infinite on that side for all time (and this happens for instance with the maximal solution constructed in [ERV]), or (b) it becomes finite at a certain time. Once a mass becomes finite (on one side) it stays finite as we show next. 
Proposition 1.1. - If the partial mass of a solution $u(x, t)$ is finite at a time $t_{1} \geq 0$, say $M_{+}\left(t_{1}\right)<\infty$, then for every $t>t_{1}$ this partial mass is finite, $M_{+}(t)<\infty$.

Proof. - We compare our solution with the maximal solution $\bar{u}$ starting at $t=t_{1}$ with the same data as $u$. For this one we multiply by a cutoff function $\zeta_{n}(x)=\zeta(n x)$ such that $0 \leq \zeta \leq 1, \zeta(x)=1$ for $x \leq 1$ and $\zeta(x)=0$ for $x \geq 2$ and integrate the equation in the rectangle $R=(0, \infty) \times\left(t_{1}, t\right)$ to get

$$
\begin{aligned}
\int_{0}^{n} u(x, t) d x \leq & \int_{0}^{\infty} \bar{u}(x, t) \zeta_{n}(x) d x=\int_{0}^{\infty} \bar{u}\left(x, t_{1}\right) \zeta_{n}(x) d x \\
& +\int_{t_{1}}^{t} \int \bar{w}(x, t) \zeta_{n, x x}(x) d x d t-\int_{t_{1}}^{t} \bar{w}_{x}(0, t) d t
\end{aligned}
$$

where $\bar{w}=\bar{u}^{m} / m$ if $m>0$ but $\bar{w}=\log (\bar{u})$ for $m=0$. We only have to control the second integral of the second member. This is done thanks to the estimate $\bar{w}=o(x)$ valid for maximal solutions, $c f$. [ERV]. This means that the integral tends to 0 as $n \rightarrow \infty$.

Combining these facts we find the following possibilities to be studied:

I. Simply infinite initial data and solution.

II. Simply infinite initial data and solution with finite mass.

III. Doubly infinite initial data and simply infinite solution.

IV. Doubly infinite initial data and solution with finite mass.

V. Doubly infinite initial data and solution.

It is clear from the results, but probably worth mentioning here, that combinations of the above cases can be produced: thus, a solution with, say, infinite mass on one side for a time $0 \leq t \leq t_{1}$, can be continued after $t_{1}$ with a finite-mass solution by prescribing a convenient flux function.

\section{UNIQUENESS OF THE SOLUTION WITH INFINITE MASS. BÄCKLUND TRANSFORM}

Our first result concerns case $\mathbf{V}$ where we have initial data which are "doubly infinite", i.e. such that

$$
\int_{-\infty}^{0} u_{0}(x) d x=\int_{0}^{\infty} u_{0}(x) d x=\infty
$$


and we want the solution $u(x, t)$ to have also doubly infinite mass for positive times, $M_{+}(t)=M_{-}(t)=\infty$. Such a solution turns out to be unique, thus giving a convenient characterization of the maximal solution under conditions (2.1).

THEOREM 1. - Let $u_{0}$ be a nonnegative and locally integrable function satisfying (2.1). Then there exists only one solution of the Cauchy Problem $(0.1)-(0.2), u \in C^{\infty}(Q), u>0$, with the property that for every $t>0$

$$
\int_{-\infty}^{0} u(x, t) d x=\int_{0}^{\infty} u(x, t) d x=\infty .
$$

This solution coincides with maximal solution constructed in [ERV].

Proof. - The existence of a maximal solution of the Cauchy problem is established in [ERV, Theorem 9]. Arguing as in Proposition 1.1 one easily concludes that infinite mass is conserved for such solutions, i.e. that $M_{+}(0)=\infty$ implies $M_{+}(t)=\infty$ for every $t>0$, and likewise for $M_{-}$.

The proof of the uniqueness result will be very different according to the value of $m$. There are two cases, $-1<m<0$ and $m=0$. In the former one we apply the technique of Bäcklund transform, BT, which allows to transform equation $(0.1)$ with exponent $m$ into the same equation with exponent $m^{\prime}=-m$. In this way the range $-1<m<0$ is mapped onto the range $0<m^{\prime}<1$. Uniqueness in the latter range is known. The BT is well-known in the case $m=-1$, where the resulting equation is the heat equation $u_{t}=u_{x x}, c f$. $[\mathrm{BK}],[\mathrm{R}],[\mathrm{H}]$. (Observe that for $m=-1$ the transform linearizes the equation; this does happen in the present case). The BT applies equation (0.1) into itself when $m=0$. The uniqueness argument is in this case more involved.

Proof of uniqueness for $m<0$. Let $u(x, t)$ be a solution of the Cauchy Problem (0.1)-(0.2) satisfying (2.1) and (2.2). We define the BT transformation as follows:

$$
\left\{\begin{array}{l}
\bar{x}(x, t)=\int_{0}^{x} u(x, t) d x+\left.\int_{0}^{t}\left(u^{m-1} u_{x}\right)\right|_{x=0} d t \\
\bar{t}=t \\
\bar{u}(\bar{x}, \bar{t})=1 / u(x, t),
\end{array}\right.
$$

which uniquely determines a function $\bar{u}(\bar{x}, \bar{t})$, solution of the problem

$$
\left\{\begin{array}{l}
\bar{u}_{t}=\left(\bar{u}^{-m-1} \bar{u}_{x}\right) \bar{x}, \quad 0 \leq-m<1 \\
\bar{u}(\bar{x}, 0)=u_{0}(\bar{x})
\end{array}\right.
$$


in the domain $\bar{I} \times(0, \infty)$ with $\bar{I}=(\bar{x}(-\infty, t), \bar{x}(\infty, t))$. Observe that the Jacobian of the transformation $\partial(\bar{x}, \bar{t}) / \partial(x, t)=u(x, t)>0$ in $Q$. Under conditions (2.2) we have $\bar{I}=\mathbf{R}$ hence the domain of $\bar{u}$ is just $Q$. Conversely, if $\bar{u}(\bar{x}, t)$ is a solution of $(\overline{P C})$ in $Q$ and we put

$$
\begin{gathered}
x=x(\bar{x}, t)=\int_{0}^{\bar{x}} \bar{u}(\bar{x}, t) d \bar{x}+\left.\int_{0}^{t}\left(\bar{u}^{-m-1} \bar{u}_{\bar{x}}\right)\right|_{\bar{x}=0} d t \\
u(x, t)=1 / \bar{u}(\bar{x}, t),
\end{gathered}
$$

then $u(x, t)$ is a solution of the original Cauchy Problem in $Q$.

Let us now check the transformation of the initial data: let

$$
M(x)=\int_{0}^{x} u_{0}(x) d x
$$

Then by the equations of the BT we have that

$$
\bar{x}(x, 0)=\int_{0}^{x} u_{0}(x) d x=M(x) .
$$

Conversely, let $\varphi(x)=M^{-1}(x)$ so that $x=\phi(\bar{x})$ (the transformation $x \rightarrow \bar{x}$ is one-to-one if $u_{0}>0$ a.e., otherwise we have to argue by limits as $t \rightarrow 0$ ). Then

$$
\bar{u}(\bar{x}, 0)=u^{-1}(x, 0)=u_{0}^{-1}(\varphi(\bar{x})) \equiv \bar{u}_{0}(\bar{x})
$$

so that

$$
M(\bar{x})=\int_{0}^{\bar{x}} \bar{u}_{0}(\bar{x}) d \bar{x}=\int_{0}^{x} \bar{u}_{0}(\bar{x}) u_{0}(x) d x=x
$$

Therefore, we conclude the equivalence of the problems:

$$
(P C)\left\{\begin{array}{l}
u_{t}=\left(u^{m-1} u_{x}\right)_{x} \\
u(x, 0)=u_{0}(x) \quad \text { con } u(x, t) \notin L_{ \pm \infty}^{1}(\mathbf{R}), \forall t \geq 0,
\end{array}\right.
$$


and

$$
(\overline{P C})\left\{\begin{array}{l}
\bar{u}_{t}=\left(\bar{u}^{-m-1} \bar{u}_{x}\right)_{\bar{x}} \\
\bar{u}(\bar{x}, 0)=\bar{u}_{0}(x) \text { with } \quad \bar{u}_{0}(x) \notin L_{ \pm \infty}^{1}(\mathbf{R}) .
\end{array}\right.
$$

By [HRP] we known that there exists a unique solution of this latter problem $\bar{u}(\bar{x}, t) \in C^{\infty}\left(((0, \infty) \times \mathbf{R}) \cap C\left([0, \infty) ; L_{\text {loc }}^{1}(\mathbf{R})\right)\right.$ and $\bar{u}(\bar{x}, t)>0$. Consequently, there exists a unique solution $u(x, t) \in$ $C^{\infty}((0, \infty) \times \mathbf{R}) \cap C\left([0, \infty) ; L_{\text {loc }}^{1}(\mathbf{R})\right)$ and $u(x, t)>0$ of the original Cauchy Problem. Obviously, it must coincide with the maximal solution constructed in [ERV].

Remark. - We can make use of the transformation even if the mass of the solution is finite. In that case the solution of the transformed problem $(\overline{P C})$ is defined in a variable spatial domain $I(t)=(\bar{a}(t), \bar{b}(t)) \equiv$ $(x(-\infty, t), \bar{x}(+\infty, t))$, with

$$
\bar{u}(\bar{a}(t), t)=\bar{u}(\bar{b}(t), t)=\infty,
$$

i.e. we get singular boundary conditions. Similarly for solutions with semi-infinite mass.

Uniqueness for $m=0$. (i) We use the same BT which now reads

$$
\left\{\begin{array}{l}
\bar{x}(x, t)=\int_{0}^{x} u(x, t) d x+\int_{0}^{t}\left(u^{-1} u_{x}\right)_{x=0} d t \\
\bar{t}=t \\
\bar{u}(\bar{x}, \bar{t})=1 / u(x, t) .
\end{array}\right.
$$

This determines a function $\bar{u}(\bar{x}, t)$ which solves problem $(\overline{P C})$ (which is the same as (PC) but for the initial function) in a domain $\bar{I}=\mathbf{R}$ as before. Therefore, a solution of problem (PC) with doubly infinite mass is mapped by BT into a similar solution of the same problem.

Claim 1. - Maximal solutions are mapped into maximal solutions.

Proof. - Let $u(x, t)$ be a maximal solution, i.e. a solution satisfying ([ERV])

$$
-\frac{u}{t} \leq u_{t} \leq \frac{u}{t}
$$


Let $\bar{u}(\bar{x}, \bar{t})$ be its image by $\mathrm{BT}$ and let

$$
\bar{v}(\bar{x}, t)=-\frac{1}{\bar{u}(\bar{x}, t)}=-u(x, t) .
$$

be the "pressure" of the image solution. Then

$$
-\bar{v}_{\overline{x x}}=u_{\overline{x x}}=\left(\frac{u_{x}}{u}\right)_{\bar{x}}=\frac{1}{u}(\log u)_{x x}=\frac{u_{t}}{u} .
$$

We conclude from the equation that

$$
\bar{v}_{\overline{x x}} \geq-\frac{1}{t} .
$$

This inequality is another way of characterizing maximal solutions, see [ERV].

(ii) We resume the proof. Let $U$ by the maximal solution of problem (PC) for a given initial function $u_{0}$ and let $u$ be any other solution. Thus, $u(x, t) \leq U(x, t)$ in $Q$. Let

$$
\bar{U}=f(\bar{X}, t) \text { and } \bar{u}=g(\bar{x}, t)
$$

be the respective transformed functions. By BT they are solutions of $(\mathrm{PC})$ in terms of the corresponding variables. They take the same initial data since the transformation is the same for both in all respects at $t=0$. Notice however that $\bar{X}$ and $\bar{x}$, as functions of $x$ and $t$, need not coincide. We know that

$$
\bar{U}(\bar{X}, t)=\frac{1}{U(x, t)} \leq \frac{1}{u(x, t)}=\bar{u}(\bar{x}, t)
$$

One has to be careful in applying this comparison since it involves in principle different space positions. We continue as follows: according to our claim the function $f(s, t)$ is a maximal solution of problem (PC) written in coordinates $s$ and $t$. Since $g(s, t)$ is also a solution we get

$$
g(s, t) \leq f(s, t) \quad \text { for every } \quad s \in \mathbf{R}, t>0 .
$$


Using (2.6) and (2.7) (with $s=\bar{x}$ ) we get

$$
\bar{U}(\bar{X}, t) \leq \bar{u}(\bar{x}, t) \leq \bar{U}(\bar{x}, t) .
$$

(iii) In order to exploit formula (2.8) we need an observation about the relative values of the functions $\bar{X}$ and $\bar{x}$. By direct differentiation of the defining formulas for fixed time we get

$$
\left.\frac{\partial \bar{X}}{\partial x}\right|_{t=\text { const }}=U(x, t),\left.\quad \frac{\partial \bar{x}}{\partial x}\right|_{t=\text { const }}=u(x, t)
$$

so that

$$
\left.\frac{\partial \bar{x}}{\partial \bar{X}}\right|_{t=c o n s t}=\frac{u}{U} \leq 1
$$

This means that for every fixed $t>0$ we can view $\bar{x}$ as a monotone increasing function of $\bar{X}$ with slope $\leq 1$. There are in principle two options:

\$ There exists at a time $t_{0}$ a point $s_{0}$ such that $\bar{x}=\bar{X}$.

In this case we use (2.8) with $X=s_{0}=\bar{x}$ to conclude that at this point $\bar{U}=\bar{u}$. In a more convenient way this means that the solutions $f$ and $g$ coincide at one point $\left(s_{0}, t_{0}\right)$. Since they are ordered solutions of equation $(0.1)$ (with $m=0$ ) and the Strong Maximum Principle can be applied, both solutions coincide, $\bar{u}=\bar{U}$. Inversing the transformation we get $u=U$.

\$ No such intersection exists.

Let us assume that $\bar{x}<\bar{X}$ for every $x \in \mathbf{R}$ and $t>0$. Using (2.8) with $y=\bar{x}$ and $z=\bar{X}$ we conclude that $z>y$ and

$$
\bar{U}(z, t) \leq \bar{u}(y, t) \leq \bar{U}(y, t)
$$

It follows that $\bar{U}$ and $\bar{u}$ are decreasing functions of their space variable, hence they have nonzero limits as $z, y \rightarrow-\infty$. The situation at $+\infty$ is studied as follows: since $z>y$ and the slope of $y^{\prime}(z) \leq 1$ there exists a constant $c$ such that

$$
z(x, t) \leq y(x, t)+c .
$$

for every $y>0$. Going back to (2.10) we find that $\bar{u}(y, t) \geq \bar{U}(y-c)$ for $y>0$. In this way we conclude that $\bar{u}$ has a decay like a maximal function on both sides. Clearly, the estimates at both ends can be done locally uniformly in $t>0$. The solution is therefore maximal, i.e. $\bar{u}=\bar{U}$. Consequently, $u=U$. A similar argument applies when $z<y$. 


\section{SIMPLY INFINITE DATA. SOLUTIONS WITH INFINITE MASS}

We begin our existence theory with the case where the initial mass is unbounded only on one side, say, as $x \rightarrow \infty$. Thus, we assume that $u_{0}$ is a nonnegative and locally integrable real function such that

$$
\int_{-\infty}^{0} u_{0}(x) d x<\infty \quad \text { and } \quad \int_{0}^{\infty} u_{0}(x) d x=\infty .
$$

According to what was said in the Section 1 we will be able to impose the usual flux data at the end $x=-\infty$. At $x=\infty$ we have two choices, either to have infinite mass at positive times, or to have finite mass. We will treat here the former situation which is mathematically simpler.

THEOREM 2. - Let $u_{0}$ be a nonnegative and locally integrable real function satisfying assumption (3.1). Let $g$ be a standard flux function. Then there exists a solution $u(x, t) \in C^{\infty}(Q) \cap C\left([0, \infty) ; L_{\mathrm{loc}}^{1}(\mathbf{R})\right)$ of problem (0.1)-(0.2) with flux data (0.4) at $x=-\infty$, while at $x=\infty$ we have $M_{+}(t)=\infty$ and

$$
u^{m}(x, t)=o(x) \text { as } x \rightarrow \infty .
$$

(For $m=0$ it is replaced by $\log (u) \geq 0(x)$.)

Remark. - Condition (3.2) is the typical condition at infinity satisfied by the maximal solutions with zero flux, see [ERV], [RV2]. Therefore, our solution is "maximal at $+\infty$ ". Our results cover the travelling waves treated in $[\mathrm{T} 1],[\mathrm{Z}]$ or $[\mathrm{COR}]$.

Proof. - We are going to obtain the solution of the Cauchy problem as the limit of the solutions of the following mixed problems

$$
\left\{\begin{array}{l}
u_{t}=\left(u^{m-1} u_{x}\right)_{x} \\
\left.\left(u^{m-1} u_{x}\right)\right|_{x=-\infty}=g(t) \\
\left.\left(u^{m-1} u_{x}\right)\right|_{x=\infty}=0 \\
u(x, 0)=u_{0, n}(x)
\end{array}\right.
$$

where $u_{0, n}$ is given by

$$
u_{0, n}(x)=u_{0}(x) \chi_{\{x \leq n\}}(x) .
$$

Vol. $12, \mathrm{n}^{\circ} 2-1995$. 
By [RV1] there exists a unique solution $u_{n}(x, t)$ of such a problem satisfying:

$$
u_{n} \in C\left([0, \infty) ; L^{1}(\mathbf{R})\right) \cap C^{\infty}(Q), u(x, t)>0, \quad \forall t>0 .
$$

By Theorem 3 of [RV1] we have

$$
\begin{gathered}
\int_{-\infty}^{+\infty}\left[u_{n}(x, t)-u_{n+1}(x, t)\right]_{+}(x, t) d x \\
\leq \int_{-\infty}^{+\infty}\left[u_{0, n}-u_{0, n+1}\right]_{+}(x) d x
\end{gathered}
$$

hence the sequence $\left\{u_{n}(x, t)\right\}$ is nondecreasing in $n$. Moreover, it is bounded above by the maximal solution corresponding to the Cauchy problem with initial data $u_{0}(x)$, hence there exists

$$
u(x, t)=\lim _{n \rightarrow \infty} u_{n}(x, t)
$$

Since the limit is taken monotonically, $u$ is positive everywhere so that by standard theory we conclude that $u(x, t) \in C^{\infty}(Q)$ and solves the equation.

The flux data are taken uniformly in $n$, and we can pass to the limit taking into account that $\left\{u_{n}^{m}(x, t)\right\}$ is a nonincreasing sequence in $n$ and

$$
\lim _{x \rightarrow \infty} u^{m-1} u_{x}(x, t)=0 \quad \Leftrightarrow \quad w=o(|x|) \quad \text { as } \quad x \rightarrow \infty
$$

with $w=u^{m} / m$ for $m<0, w=\log (u)$ for $m=0$.

TheOREM 3. - The solution of Theorem 2 is unique.

Proof. - Let us check that the solution $u$ constructed in Theorem 2 is unique. For that let $v(x, t)$ be another solution of the problem. Let

$$
w=\left(u^{m}-v^{m}\right) / m, \quad m>0, \quad(w=\log (u / v) \quad \text { for } \quad m=0),
$$

let $\phi \in C^{\infty}(\mathbf{R})$ such that $0 \leq \phi \leq 1, \phi(x)=1$ for $x \leq 1$ and $\phi(x)=0$ for $x \geq 2$, we define $\phi_{n}$ by $\phi_{n}(x)=\phi(x / n)$ and choose a function 
$p \in C^{\infty}(\mathbf{R})$ such that $p(s)=0$ for $s<0,0<p^{\prime}(s)<1$ for $s>0$ and $p(\infty)=1$. Then, for every fixed $t>0$ we have

$$
\begin{aligned}
\int_{-n}^{\infty} \phi_{n}(x) p(w)(u-v)_{t} d x= & -\int w_{x}\left(p(w) \phi_{n}\right)_{x} d x-\left.w_{x} p(w)\right|_{x=-n} \\
& \leq-\left.w_{x} p(w)\right|_{x=-n}-\int_{-n}^{\infty} w_{x} p(w) \phi_{n x} \\
& =-\left.w_{x} p(w)\right|_{x=-n}+\int_{-n}^{\infty} j(w) \phi_{n, x x} d x
\end{aligned}
$$

where $j(s)=\int_{0}^{s} p(r) d r$. Letting $p(r) \rightarrow \operatorname{sign}_{+}(r)$ we have $j(s) \rightarrow[s]_{+}$ and then

$\frac{d}{d r} \int_{-n}^{\infty}[u(x, t)-v(x, t)]_{+} \phi_{n}(x) \leq-\frac{d}{d x}[w]_{+}(-n, t)+\int_{n}^{2 n}[w]_{+} \phi_{n, x x} d x$

Integrating in $0 \leq s<t$ we have

$$
\begin{aligned}
& \int_{-n}^{\infty}[u(x, t)-v(x, t)]_{+} \phi_{n}(x) d x \\
& \quad \leq \int_{-n}^{\infty}[u(x, s)-v(x, s)]_{+} \phi_{n}(x) d x \\
& \quad-\int_{s}^{t}[w]_{x}^{+}(-n, \tau) d \tau+\int_{s}^{t} \int_{n}^{2 n}[w]_{+} \phi_{n, x x} d x d \tau .
\end{aligned}
$$

Letting $n \rightarrow \infty$ and taking into account that $[w]_{+}=o(x)$ as $x \rightarrow \infty$ and the flux condition at $x=-\infty$ we end the proof.

\section{SIMPLY INFINITE DATA. SOLUTIONS WITH FINITE MASS}

We tackle now the case where as in the previous section we have initial data with infinite mass on one end but now we want to construct a solution with finite mass on both ends for all positive times $t>0$. We recall that the existence of such solutions is new and makes this theory very different from the classical heat equation, and more generally from equation (0.1) with $m>0$. 
In view of the mass balance, formula (1.1), the flux function must have infinite integral at $t=0$. This turns out to be the only extra condition. The existence of the solution is based on approximation using the existence results of [RV1]. A novel feature is the fact that we can also prescribe the mass at any positive time, say at $t=1$. The precise result is as follows.

THEOREM 4. - Let $u_{0}$ be a nonnegative and locally integrable real function satisfying (3.1). Let $f$ be a nonnegative flux function in $B V_{\mathrm{loc}}(0, \infty)$ such that $\int f(t) d t$ diverges at $t=0$ and let $g$ be a standard fux function. Finally, let $t_{0}$ be any positive time and let $M$ be a number, $0 \leq M<\infty$. Then there exists a solution of the mixed problem (0.1)-(0.4) defined for $0<t<t_{0}$ and satisfying moreover

$$
\int_{-\infty}^{+\infty} u\left(x, t_{0}\right) d x=M
$$

Proof. - (i) For simplicity we take without loss of generality $t_{0}=1$. We obtain the solution of the Cauchy problem as the limit of the solutions of the following mixed problems

$\left(\mathrm{MP}_{n}\right)$

$$
\left\{\begin{array}{l}
u_{t}=\left(u^{m-1} u_{x}\right)_{x} \\
\lim _{x \rightarrow+\infty}\left(u^{m-1} u_{x}\right)(x, t)=-f_{n}(t) \\
\lim _{x \rightarrow-\infty}\left(u^{m-1} u_{x}\right)(x, t)=g(t) \\
u(x, 0)=u_{0, n}(x)
\end{array}\right.
$$

where we define

$$
\begin{gathered}
u_{0, n}(x)=u_{0}(x) \chi_{\{x \leq n\}}(x), \\
f_{n}(t)=f(t) \chi_{\left\{t \geq \varepsilon_{n}\right\}}(t),
\end{gathered}
$$

$\chi_{E}$ denoting the characteristic function of a set $E ; \varepsilon_{n}$ is a positive and nonincreasing sequence chosen so that the following mass balance is satisfied:

$$
\int_{0}^{1}\left(f_{n}+g\right) d t+M=\int_{-\infty}^{\infty} u_{0, n}(x) d x
$$


In other words, we set

$$
\left\{\begin{array}{c}
\int_{\varepsilon_{n}}^{1} f(t) d t=\int_{0}^{n} u_{0}(x) d x-M_{1} \\
M_{1}=\int_{0}^{1} g d t+M-\int_{-\infty}^{0} u_{0}(x) d x .
\end{array}\right.
$$

This is where the divergence of $f$ is needed. Clearly, $\varepsilon_{n} \rightarrow 0$ as $n \rightarrow \infty$. For every $n$ problems $\left(M P_{n}\right)$ satisfies the conditions of Theorem 2 of [RV1], hence there exists a solution $u_{n}(x, t)$ satisfying:

$$
\left\{\begin{array}{l}
u_{n}(x, t) \in C\left([0, \infty) ; L^{1}(\mathbf{R})\right) \cap C^{\infty}((0, \infty) \times \mathbf{R}) \\
u_{n}(x, t)>0, \quad \forall t>0, \quad \forall x \in \mathbf{R} .
\end{array}\right.
$$

Moreover, the mass balance at time $0<t<1$ reads

$$
\begin{aligned}
M_{n}(t)=\int_{-\infty}^{+\infty} u_{n}(x, t) d x & =\int_{-\infty}^{+\infty} u_{0, n}(x) d x-\int_{0}^{t}\left(f_{n}+g\right) d t \\
& =M+\int_{t}^{1}\left(f_{n}+g\right) d t
\end{aligned}
$$

It follows that

$$
\int_{-\infty}^{+\infty} u_{n}(x, 1) d x=\int_{-\infty}^{n} u_{0}(x) d x-\int_{0}^{1}\left(f_{n}+g\right) d t=M
$$

holds independently of $n$. On the other hand, if $0<\varepsilon_{n}<t \leq 1$ we get for $t>0$ as a consequence of (4.4)

$$
\int_{-\infty}^{\infty} u_{n}(x, t) d x \leq \int_{-\infty}^{\infty} u_{n+1}(x, t) d x, \quad \forall n .
$$

(ii) We now consider the potential functions:

$$
U_{n}(x, t)=\int_{-\infty}^{x} u_{n}(x, t) d x+\int_{0}^{t} g(t) d t
$$

Since the $u_{n}(x, t)$ are solutions of equation $(0.1)$, this expression can be written equivalently in the form:

$$
U_{n}(x, t)=\int_{-\infty}^{x} u_{0, n}(x) d x+\int_{0}^{t}\left(u_{n}^{m-1} u_{x}\right)(x, t) d t
$$


The $U_{n}(x, t)$ are the unique solutions of the problems

(P)

$$
\left\{\begin{aligned}
U_{t}=\left|U_{x}\right|^{m-1} U_{x x} & \\
U(+\infty, t) & =\int_{-\infty}^{+\infty} u_{n}(x, t) d x+\int_{0}^{t} g d t \\
& =\int_{-\infty}^{+\infty} u_{0, n} d x-\int_{0}^{t} f_{n} d t \\
U(-\infty, t) & =\int_{0}^{t} g(t) d t, \\
U(x, 0) & =\int_{-\infty}^{x} u_{0, n}(x) d x .
\end{aligned}\right.
$$

Observe that we can write the value of $U_{n}(\infty, t)$ as

$$
U_{n}(\infty, t)=M+\int_{t}^{1} f_{n}(t) d t+\int_{0}^{1} g(t) d t
$$

which is independent of $n$ for $t \geq \varepsilon_{n_{0}}, n \geq n_{0}$. In view of the conditions we have $U_{n}(x, 0) \leq U_{n+1}(x, 0), U_{n}(-\infty, t)=U_{n+1}(-\infty, t)$, $U_{n}(+\infty, t) \leq U_{n+1}(+\infty, t)$ and the Maximum Principle we obtain $U_{n} \leq U_{n+1}$, hence

$$
\int_{-\infty}^{x} u_{n}(x, t) d x \leq \int_{-\infty}^{x} u_{n+1}(x, t) d x
$$

Consequently, $U_{n}(x, t)$ is a nondecreasing sequence. Now, if we consider the solution $\bar{u}(x, t)$ corresponding to the mixed problem with initial data $u_{0}(x)$ and flux data zero at $+\infty$ and the same $g$ at $-\infty$, as constructed in Theorem 2, we have for all $n$

$$
u_{n}(x, t) \leq \bar{u}(x, t)
$$

so that

$$
U_{n}(x, t) \leq V(x, t)
$$


where $V(x, t)$ the integral of the solution $\bar{u}(x, t)$, defined as in (4.6). Hence, there exists

$$
U(x, t)=\lim _{n \rightarrow \infty} U_{n}(x, t)
$$

(iii) We now check that $u(x, t)=\partial U / \partial x>0$ everywhere, which implies that $U(x, t)$ is the solution of the problem:

$$
\left\{\begin{array}{l}
U_{t}=\left|U_{x}\right|^{m-1} U_{x x} \\
U(-\infty, t)=\int_{0}^{t} g d t \\
U(+\infty, t) \int_{0}^{1} g d t+M+\int_{t}^{1} f d t \\
U(x, 0)=\int_{-\infty}^{x} u_{0}(x) d x
\end{array}\right.
$$

and, correspondingly, $u(x, t)$ satisfies the conditions of Theorem 4. Due to the fact that $f_{n} \in B V(0, \infty)$ we can apply Lemma 5.7 of [RV1] to conclude that

$$
\left|\left(u_{n}^{m}\right)_{x}(x, t)\right| \leq \frac{K}{t}, \quad \forall t \geq \varepsilon>0
$$

independently of $u$. Letting $n \rightarrow \infty$ :

$$
\left|\left(u^{m}\right)_{x}(x, t)\right| \leq \frac{K}{t}
$$

which implies that either $u(x, t) \equiv 0$, or $u(x, t)>0$, for all $t \geq \varepsilon>0$. Now, the first alternative is not possible for $t<1$ since

$$
U(-\infty, t)=\int_{0}^{t} g(t) d t
$$

and

$$
U(+\infty, t) \geq U_{n_{0}}(+\infty, t)=M+\int_{0}^{1} g(t) d t+\int_{t}^{1} f(t) d t
$$

so that $U(\infty, t)-U(-\infty, t)>M$. Thus, $u$ cannot be identically zero. Notice that $u(\cdot, t) \in L^{1}(-\infty, 0]$.

Vol. 12, $n^{\circ} 2-1995$. 
(iv) Finally, we check that the initial data $u_{0}(x)$ is taken uniformly in $L_{\text {loc }}^{1}(\mathbf{R})$ as $t \rightarrow 0$. Let $R$ fixed and $n_{0}$ large enough. Then

$$
\begin{aligned}
\int_{-\infty}^{R} u(x, t) d x & \geq \int_{-\infty}^{R} u_{n_{0}}(x, t) d t \\
& \geq \int_{-\infty}^{R} u_{0}(x) d x-\varepsilon
\end{aligned}
$$

where we have used (4.8) and the fact that $u_{n} \in C\left([0, \infty) ; L^{1}(\mathbf{R})\right)$. On the other hand, if we take the maximal solution $\bar{u}(x, t)$ :

$$
\int_{-\infty}^{R} u(x, t) d x \leq \int_{-\infty}^{R} \bar{u}(x, t) d t \leq \int_{-\infty}^{R} u_{0}(x) d x+\varepsilon .
$$

Hence, $\int_{-\infty}^{R} u(x, t) d x \rightarrow \int_{-\infty}^{R} u_{0}(x) d x$, uniformly if $0<t<\varepsilon$.

Remarks. - 1) If $M>0$ the solution can be continued beyong $t=t_{0}$ as a finite mass solution by prescribing acceptable flux data at $\pm \infty$ and using the existence and uniqueness theory of [RV1].

2) The above construction gives for every fixed triple $\left(u_{0}, f, g\right)$, and fixing also $t_{0}>0$, a one-parameter family of different solutions, with parameter $M \geq 0$. It is clear that $t_{0}$ is not an additional freedom of the set of solutions since changing the time $t_{0}$ does not produce an essentially different family. To be precise, changing from $t_{0}=1$ to $t_{0}<1$ only gives as additional solutions those with maximal time $t_{0}$ (i.e. $M\left(t_{0}\right)=0$ ) which cannot of course be continued beyong $t_{0}$.

We have been unable to settle the uniqueness of the solution constructed with the above specifications (viz $u_{0}, g, f, t_{0}$ and $M$ ). However we have the following characterization.

THEOREM 5. - The solution constructed above is the minimal solution in an integral sense among the solutions of the stated problem.

Proof. - Let $u(x, t)$ the solution just constructed and let $v(x, t)$ be any other solution of the problem with the same data. We compare the problems satisfied by the integrated functions, $U_{n}(x, t)$ defined in (4.6) and the corresponding formula for $V(x, t)$ in terms of $v$. It is immediate from the Maximum Principle that $U_{n}(x, t) \leq V(x, t)$, hence in the limit $U(x, t) \leq V(x, t)$, i.e. $U(x, t)$ is minimal. 


\section{INFINITE MASS ON BOTH SIDES}

We turn now to the study of solutions whose initial data are infinite on both ends, i.e. when

$$
\int_{0}^{\infty} u_{0}(x) d x=\int_{-\infty}^{0} u_{0}(x) d x=\infty
$$

We begin with the case where we impose a flux function on only one side, the other side being assigned zero flux.

THEOREM 6. - Let $u_{0}$ be a nonnegative and locally integrable function satisfying (5.1). Let $f \in B V_{\mathrm{loc}}(0, \infty)$ be a nonnegative flux function such that $\int_{0}^{t} f(t) d t=\infty$. Let also $t_{0}>0$ and $M_{1} \in \mathbf{R}$. Then there exists $a$ solution of the Cauchy Problem such that for all $t>0$

$$
\int_{-\infty}^{0} u(x, t) d x=\infty \quad \text { and } \quad \int_{0}^{\infty} u(x, t) d x<\infty,
$$

satisfying also

$$
\left.\int_{0}^{t_{0}}\left(u^{m-1} u_{x}\right)\right|_{x=0} d t+\int_{0}^{\infty} u\left(x, t_{0}\right) d x=M_{1} .
$$

Proof. - (i) As before we take $t_{0}=1$. Here we are going to obtain the solution $u(x, t)$ as the limit of the solutions of the following mixed problems

(PM)

$$
\left\{\begin{array}{l}
u_{t}=\left(u^{m-1} u_{x}\right)_{x} \\
u^{m-1} u_{x}(-\infty, t)=0 \\
-u^{m-1} u_{x}(+\infty, t)=f(t) \\
u(x, 0)=u_{0, n}(x)
\end{array}\right.
$$

where now $u_{0, n}(x)=u_{0}(x)$ if $x \geq-n$ and 0 for $x<-n$. Thanks to Theorem 4 (after the change $x \rightarrow-x$ ) for every $n$ there exists a solution $u_{n}(x, t)$ satisfying the standard properties. We also want (5.3). This is obtained as follows:

$$
\begin{aligned}
\int_{0}^{1} & \left.u_{n}^{m-1} u_{n, x}\right|_{x=0} d t+\int_{0}^{\infty} u_{n}(x, 1) d x \\
\quad= & \int_{0}^{-\infty} u_{0, n}(x) d x+\int_{-\infty}^{\infty} u_{n}(x, 1) d x=I_{1}+I_{2} .
\end{aligned}
$$


Since we can fix $I_{2}$ at any value $M_{n} \geq 0$ and $I_{1} \rightarrow-\infty$ as $n \rightarrow \infty$ for all $n$ large enough we can obtain a solution with the property (5.3).

(ii) We now define the integral function

$$
U_{n}(x, t)=\left.\int_{0}^{t}\left(u_{n}^{m-1} u_{n, x}\right)\right|_{x=0} d t+\int_{0}^{x} u_{n}(x, t) d x
$$

which is solution of the following problem:

$$
\left\{\begin{aligned}
U_{t}=\left|U_{x}\right|^{m-1} U_{x x} & \\
U(-\infty, t) & =-\int_{-\infty}^{0} u_{n}(x, t) d x+\left.\int_{0}^{t}\left(u_{n}^{m-1} u_{n, x}\right)\right|_{x=0} d t \\
& =\int_{0}^{-\infty} u_{0, n} d x \\
U(+\infty, t) & =\int_{0}^{\infty} u_{n}(x, t) d x+\left.\int_{0}^{t}\left(u_{n}^{m-1} u_{n, x}\right)\right|_{x=0} d t \\
& =M_{1}+\int_{t}^{1} f(t) d t \\
U(x, 0) & =\int_{0}^{x} u_{0, n}(x) d x
\end{aligned}\right.
$$

The sequence $\left\{U_{n}(x, t)\right\}$ satisfies the following conditions:

$$
\left\{\begin{array}{l}
U_{n}(+\infty, t)=\text { constant with } n \\
U_{n}(x, 0)=\int_{0}^{x} u_{0, n}(x) d x \geq U_{n+1}(x, 0) \\
U_{n}(-\infty, t)=U_{n}(-\infty, 0) \geq U_{n+1}(-\infty, 0) .
\end{array}\right.
$$

We may apply the Maximum Principle and obtain

$$
U_{n}(x, t) \geq U_{n+1}(x, t), \quad \forall n .
$$

(iii) We want to establish the convergence of the monotone sequence $U_{n}$. For this we only need a bound from below and this is given by the solution $\bar{u}_{m}$ of problem $(P M)$ with initial data

$$
\bar{u}_{m}(x, t)= \begin{cases}u_{0}(x) & \text { for } x \leq m \\ 0 & \text { otherwise }\end{cases}
$$


zero flux data at $x=-\infty$, and finite flux data at infinity:

$$
\bar{f}_{m}(t)= \begin{cases}f(t) & \text { for } \quad \varepsilon_{m} \leq t \leq 1 \\ 0 & \text { for } \quad 0<t<\varepsilon_{m}\end{cases}
$$

where $m$ is large and $\varepsilon_{m}$ is determined by the condition

$$
\int_{0}^{m} u_{0}(x) d x=\int_{\varepsilon_{m}}^{1} f(t) d t+M_{1}
$$

(this needs $m \gg 1$ ), so that the corresponding integrated function $\bar{U}_{m}$ given by (5.4) still satisfies $\bar{U}_{m}(\infty, t)=M_{1}+\int_{t}^{1} f(t) d t$ for $\varepsilon_{m} \leq t \leq 1$, while $\bar{U}_{m}(\infty, t)=\bar{U}_{m}(\infty, 0)$ for $0<t<\varepsilon_{m}$. The existence of such a solution comes from Theorem 2 above.

It is clear from the boundary conditions that $\bar{U}_{m}(x, t) \leq U_{n}(x, t)$ for every $n, m>0$ large enough. Hence we can pass to the limit

$$
\lim _{n \rightarrow \infty} U_{n}(x, t)=U(x, t) .
$$

On the other hand, since $\left|\left(u_{n}^{m-1} u_{n, x}\right)(x, t)\right| \leq c / t$, either we have that $u>0$ or $u \equiv 0, \forall t>\varepsilon>0$. As $u \equiv 0$ would imply that $u(x, t)=0$ and in the limit $U(-\infty, t)=-\infty$ while $U(\infty, t)$ is finite, we conclude that $u>0$.

(iv) Moreover, we have

$$
\int_{0}^{\infty} u_{n}(x, t) d x=M+\int_{t}^{1} f-\int_{0}^{t}\left(u_{n}^{m-1} u_{n, x}\right)(0, t) d t
$$

Letting $n \rightarrow \infty, t \geq \varepsilon>0$ :

$$
\int_{0}^{\infty} u(x, t) d x \leq k
$$

If we now consider:

$$
\begin{aligned}
\int_{-\infty}^{0} u_{n}(x, t) d x= & \int_{-\infty}^{0} u_{0, n}(x) d x+\int_{0}^{t} u_{n}^{m-1} u_{n, x}(0, t) d t \\
& \geq \int_{-\infty}^{0} u_{0 n}(x) d x-C
\end{aligned}
$$


Letting $n \rightarrow \infty$, if $t \geq \varepsilon>0$ we have that $\int_{-\infty}^{0} u(x, t) d x=\infty$. Therefore, $u \notin L^{1}(-\infty, R)$ and since $u_{n}^{m-1} u_{x} \rightarrow 0$ as $x \rightarrow-\infty \forall n$, we have that

$$
u^{m}=o(|x|) \text { if } \quad x \rightarrow-\infty .
$$

The proof is complete.

\section{SOLUTIONS WITH FINITE MASS FOR DOUBLY INFINITE DATA}

THEOREM 7. - Let $u_{0}$ be a nonnegative, nontrivial and locally integrable function satisfying $\int_{-\infty}^{0} u_{0}(x) d x=\int_{0}^{\infty} u_{0}(x) d x=\infty$. Let $f(t)$ and $g(t)$ be nonnegative flux functions in $B V_{\mathrm{loc}}(0, \infty)$ both with diverging integrals at $t=0$. Let $t_{0}$ be a fixed time $>0$ and let $M, M_{1}$ be two constants, $M \geq 0$ and $M_{1} \in \mathbf{R}$. Then there exists a solution of $(0.1)-(0.4)$ such that

$$
\int u\left(x, t_{0}\right) d x=M<\infty .
$$

and

$$
\left.\int_{0}^{t_{0}} u^{m-1} u_{x}\right|_{x=0} d t+\int_{0}^{\infty} u\left(x, t_{0}\right) d x=M_{1}
$$

Proof. - As before we may take $t_{0}=1$. We approximate $u_{0}$ by $u_{0, n}=u_{0} \cdot \chi_{[-n,+\infty)}$. We solve the problems

$$
\left\{\begin{array}{l}
u_{t}=\left(u^{m-1} u_{x}\right)_{x} \\
u(x, 0)=u_{0 n}(x) \\
\left(u^{m-1} u_{x}\right)(+\infty, t)=-f(t) \\
\left(u^{m-1} u_{x}\right)(-\infty, t)=g_{n}(t),
\end{array}\right.
$$

where $g_{n}(t)=g(t) \cdot \chi_{\left[\varepsilon_{n}, 1\right)}(t)$ with $\varepsilon_{n}$ determined through the condition

$$
\int_{0}^{-\infty} u_{0 n}(x) d x+\int_{0}^{1} g_{n}(t) d t=M_{2}
$$

with $M_{2}=M_{1}-M$. Observe that $\varepsilon_{n} \rightarrow 0$ and the $g_{n}$ 's form a nondecreasing sequence. By Theorem 4 above there exists a solution 
$u_{n}(x, t)>0, u_{n} \in C\left([0, \infty): L_{\mathrm{loc}}^{1}(\mathbf{R})\right)$ of this problem satisfying the extra condition

$$
\int_{-\infty}^{\infty} u_{n}(x, 1) d x=M<\infty
$$

We define the integrated functions $U_{n}(x, t)$ as in (5.4). They satisfy a similar problem. In particular the value at $x=\infty, 0<t<1$ is independent of $n$, while the value at $x=-\infty$

$$
U_{n}(-\infty, t)=M_{2}-\int_{t}^{1} g_{n}(t) d t
$$

is nonincreasing with $n$. An application of the Maximum Principle gives $U_{n+1}(x, t) \leq U_{n}(x, t)$. Since $U_{n}(x, t)$ is monotone nondecreasing in $x$ for every $t$ we conclude that there exists the limit

$$
\lim _{n \rightarrow \infty} U_{n}(x, t)=U(x, t),
$$

and $U(x, t) \leq U_{n}(x, t)$.

We have to verify that $u=\partial U / \partial x$ is a solution of our problem, or equivalently that $U$ is a solution of the integrated problem. First of all, $U$ satisfies obviously the boundary condition at $x=-\infty$ (use the fact that it is a monotone limit of monotone functions). As for the limit at $x=\infty$ it follows by comparison with the solution constructed in Theorem 6 , which lies obviously below (compare the boundary data). Both boundary conditions mean immediately (by arguments already used) that $u$ is a no trivial solution of $(0.1)$ and that the initial data are satisfied.

Remark. - As mentioned in Section 4 the solution constructed here for $0<t<t_{0}$ can be continued if $M>0$ with the standard theory for $L^{1}$ data, $c f .[\mathrm{RV} 1]$.

\section{CONCLUSION AND COMMENTS}

We have discussed the existence of multiple solutions for the Cauchy problem (0.1)-(0.2) with initial data $u_{0} \in L_{\text {loc }}^{1}(\mathbf{R}), u_{0} \geq 0, u_{0}$ nontrivial.

Our main point has been to show that, independently of the bounded or unbounded character of the mass function $\int_{0}^{x} u_{0}(x) d x$ as $x \rightarrow \pm \infty$, there is always the possibility of adjoining suitable flux functions on both ends, (0.3)-(0.4), thus giving rise to an infinite multiplicity of solutions 
parametrized by the functions $f$ and $g$. Moreover, we have also found an additional free parameter in the solution set for problems with infinite mass on one end at $t=0$ but finite mass for $t>0$ (two free parameters if the above situation occurs at both ends). The behaviour of the solutions of $x=\infty$ and $x=-\infty$ has turned out to be independent of each other in the matters of concern in this study.

As for the characterization of the solutions we have shown the uniqueness of the solutions corresponding to situations at $\pm \infty$ of the form: (i) infinite mass for all times (without any further specification), or (ii) finite mass for all times (with flux condition). The uniqueness of the solutions with infinite initial mass but finite mass for $t>0$ is open (recall that these are the cases with additional parameters).

Observe also the behaviour of the solutions as $|x| \rightarrow \infty$. If the flux, say $f(t)$ is not zero then $u \rightarrow 0$ as $x \rightarrow \infty$ with the rate

$$
u \sim x^{1 / m} \quad \text { if } \quad m<0, \quad u \sim e^{-c x} \quad \text { if } \quad m=0,
$$

same behaviour at $-\infty$. When the flux is zero we get $1 / u \leq O\left(|x|^{2 /(1-m)}\right)$, cf. [ERV].

We are talking about non-uniqueness of positive solutions to a Cauchy problem, something that does not happen for $m>0$. Moreover, since the functions $u \equiv$ constant are maximal solutions (Theorem 1), the infinitely many solutions corresponding to initial data $0 \leq u_{0}(x) \leq c$ are bounded and satisfy all of them $0 \leq u(x, t) \leq c$.

It is worth mentioning the change of variables $w=u^{m}(m<0)$ which transforms equation $(0.1)$ into

$$
w_{t}=w^{\alpha} w_{x x}, \quad \alpha=(m-1) / m>0 .
$$

Then $0<u<c$ implies $\infty>w>C=c^{1 / m}$. In the case $m=0$ if we do $w=-\log (u)$ the equation becomes

$$
w_{t}=e^{w} w_{x x} .
$$

With these definitions $w \rightarrow+\infty$ as $|x| \rightarrow \infty$ in both cases for solutions with nonzero flux.

Though the data considered here are quite general, there are natural extensions. Thus, the initial data can be taken in the set of locally bounded measures. The corresponding study for maximal solutions is done in [RV2]. Adapting our results to such generality involves few essential novelties and some technical complications, that we have preferred to avoid. Possibly 
more interesting is the generalization of the flux data (also to nonnegative measures), where some curious results appear.

The features described in the present analysis apply to other boundary value problems for equation (0.1) in the same exponent range $0 \geq m>-1$. This is in particular true of the mixed problem posed in a quarter of the plane, $Q=(0, \infty) \times(0, \infty)$, with Neumann data at $x=0$. The analysis is essentially the same, being simplified by the fact that we deal with only one free end, $x=\infty$. For this reason it could even be preferrable for expository purposes.

Let us finally remark that the above described situation is not found when we consider equation $u_{t}=\operatorname{div}\left(u^{m-1} D u\right)$ in several space dimensions, $N>1$, with $m \leq 0$. Thus, it is proved in [V] that finite mass solutions posed in a domain $Q=\mathbf{R}^{N} \times(0, T)$ exist only for $N=2$ and $m=0$. Even in this case the results are different, $c f$. [VER].

\section{REFERENCES}

[BK] G. BLUMAN and S. KuMEI, On the remarquable nonlinear diffusion equation $\partial / \partial x\left[a(u+b)^{-2}(\partial u / \partial x)\right]-(\partial u / \partial t)=0$, J. Math. Phys., Vol. 21, 1980 pp. 1019-1023.

[C] T. Carleman, Problèmes mathématiques dans la théorie cinétique des gaz, Almqvist and Wilsell, Uppsala, 1957.

[COR] T. Chayes, S. J. Osher and J. V. Ralston, On singular diffusion equations with applications to selforganized criticality, preprint.

[DGZ] V. van DUIN, S. GoMEs and H. F. ZHANG, On a class of similarity solutions of the equation $u_{t}=\left(u^{m-1} u_{x}\right)_{x}$ with $m>-1$, IMA J. Appl. Math., VoI. 41, 1988, pp. 147-163.

[ERV] R. Esteban, A. Rodríguez and J. L. VAZqueZ, A nonlinear heat equation with singular diffusivity, Comm. Partial Diff. Equations, Vol. 13, 1988, pp. 985 1039.

[H] M. A. Herrero, A limit case in nonlinear diffusion, Nonlinear Anal., Vol. 13, 1989, pp. 611-628.

[HP] F. HellfFerich and M. C. Plesset, Ion exchange kinetics: a nonlinear diffusion problem, J. Chem. Phys., Vol. 28, 1958, pp. 418-424.

[HRP] A. Herrero and M. PierRe, The Cauchy problem for $u_{t}=\Delta u^{m}$ when $0<m<1$, Trans. Amer. Math. Soc., Vol. 291, 1985, pp. 145-158.

[LH] K. E. Longren and A. HiRose, Expansion of an electron cloud, Phys. Letters A, Vol. 59, 1976, pp. 285-286.

[MK] H. P. McKEAN, The central limit theorem for Carleman's equation, Israel Jour. Math., Vol. 21, 1976, pp. 54-92.

[R] G. Rosen, Nonlinear heat conduction in solid $H_{2}$, Phys. Rev. B, Vol. 19, 1979, pp. 2398-2399.

[RV1] A. RodRiguez and J. L. VAZQUEZ, A well posed problem in singular Fickian diffusion, Arc. Rat. Mech Anal., Vol. 110, 1990, pp. 141-163.

[RV2] A. RodRíguez and J. L. VAZQUEZ, Maximal solutions of singular diffusion equations with general initial data, in "Nonlinear Diffusion Equations and Their Equilibrium States", 3, N. G. LLoyd et al. eds, Birkhäuser, 1992, pp. 471-484. 
[T1] P. TAKÁČ, A fast diffusion equation which generates a monotone local semiflow I: local existence and uniqueness, Diff. Int. Eqns.

[T2] P. TAKÁČ, A fast diffusion equation which generates a monotone local semiflow II: global existence and asymptotic behaviour, Diff. Int. Eqns.

[V] J. L. VAZQUEZ, Nonexistence of solutions for nonlinear heat equations of fastdiffusion type, J. Math. Pures Appl., Vol. 71, 1992, pp. 503-526.

[VER] J. L. VazQuez, J. R. Esteban and A. Rodríguez, The fast diffusion equation with logarithmic nonlinearity and the evolution of conformal metrics in the plane, preprint, UAM Madrid, 1994.

[Z] H. F. ZHANG, A nonlinear singular diffusion problem: existence and uniqueness, Applicable Analysis, to appear.

(Manuscript received November 25, 1993; accepted February 28, 1994.) 